\title{
A 57-year-old chronic cougher with somatically evoked cough
}

\author{
Federico Lavorini, Giovanni A. Fontana* \\ Department of Experimental and Clinical Medicine, University of Florence, Florence Italy
}

\section{A R T I C L E I N F O}

\section{Article history:}

Received 16 March 2017

Received in revised form

25 May 2017

Accepted 26 May 2017

Available online 28 May 2017

\section{Introduction}

Chronic cough is one of the most common symptoms of medical importance [1]. The differential diagnosis of chronic cough is extensive [2]. Uncommon causes of chronic cough may be missed unless an adequate history is obtained, a detailed examination performed and appropriate investigations arranged [3]. Herein, we present a case of chronic cough with some peculiar features that were previously unreported.

\section{Case presentation}

A 57-year-old man presented with chronic non-productive cough over a 14-month period. He was a non-smoker, nonallergic subject, and had no history of environmental exposure to chemicals or dust. The cough was non-seasonal, both diurnal and nocturnal, and worsened by strong smells or perfumes; in addition, it was often evoked by unusual events such as tying a tie, rubbing his neck or even when his neck or thorax was exposed to an air stream when undressed. His medical history was unremarkable, except from hypothyroidism requiring thyroid replacement therapy, typical [4] symptoms of gastro-oesophageal reflux, intermittent sneezing and nasal obstruction. The patient did not complain of post-nasal drip. During the past 1.5 years, he had undergone to a series of examinations that revealed no lung function impairment (forced expiratory volume at $1 \mathrm{~s}$ : $4.55 \mathrm{~L}$ 134\% predicted, slow vital capacity: 6.22 L, 139\% predicted, functional residual capacity: $3.34 \mathrm{~L}$ 94\% predicted) and normal lung parenchyma on chest radiograph. Laryngeal endoscopy revealed inter-arytenoid oedema. The skull CT scan was unremarkable. On admission, the cough was reportedly

\footnotetext{
* Corresponding author. Department of Experimental and Clinical Medicine, University of Florence, Italy, Largo Brambilla, 3, 50134 Firenze, Italy.
}

E-mail address: giovanni.fontana@unifi.it (G.A. Fontana). unaffected by proton-pump inhibitor therapy and by antacid intake. The patient was a tall, overweight man (body mass index 35.51) with normal vital signs. Oropharyngeal examination revealed a hyperaemic oropharynx. Chest examination was normal and he had no peripheral oedema, clubbing, or cyanosis. He suffered from a tender upper abdomen and coughing upon maximal lung emptying (deflation cough) [5-7]. Coughing could consistently be evoked also by digital pressure along the sternal bone, particularly the lower third toward the xiphoidal process, along both sides of the neck anterior to the sternocleidomastoid muscle, and on the cervical or first dorsal vertebrae. Although not formally assessed, the number of the evoked cough efforts appeared to be highly variables depending upon the region involved and the pressure exerted. The cough frequency also varied between trials. A representation of the body areas from which the cough could be evoked by digital pressure is schematized in Fig. 1. In all instances, the onset of an urge to cough (UTC) preceded cough appearance. The patient rated the intensity of the UTC from 4/9 to 6/9 using a visual analogue scale. Notably, both the UTC and cough were temporarily (about $30 \mathrm{~s}$ ) abolished by prior topical application of dry ice on the relevant skin areas. The patient was discharged with prescriptions of nasal wash and topic steroids, ranitidine $300 \mathrm{mg}$ b.i.d. and life-style recommendations. On a second consultation two months later, the patient reported that the cough was largely unchanged, though he felt a slight improvement in both nasal and reflux symptoms. In such occasion we observed that UTC and coughing could be evoked by the same manoeuvres as those described above. Manipulation of the anterior region of the neck was ineffective. The patient was then prescribed esomeprazole (40 mg bid), metoclopramide (10 mg tid) and antacids. Nasal wash was maintained. On a subsequent visit, about three months later, the patient reported a marked improvement of the cough. Notably, digital pressure along the neck, sternum and spine were still effective in evoking cough, albeit to a much lesser extent. 

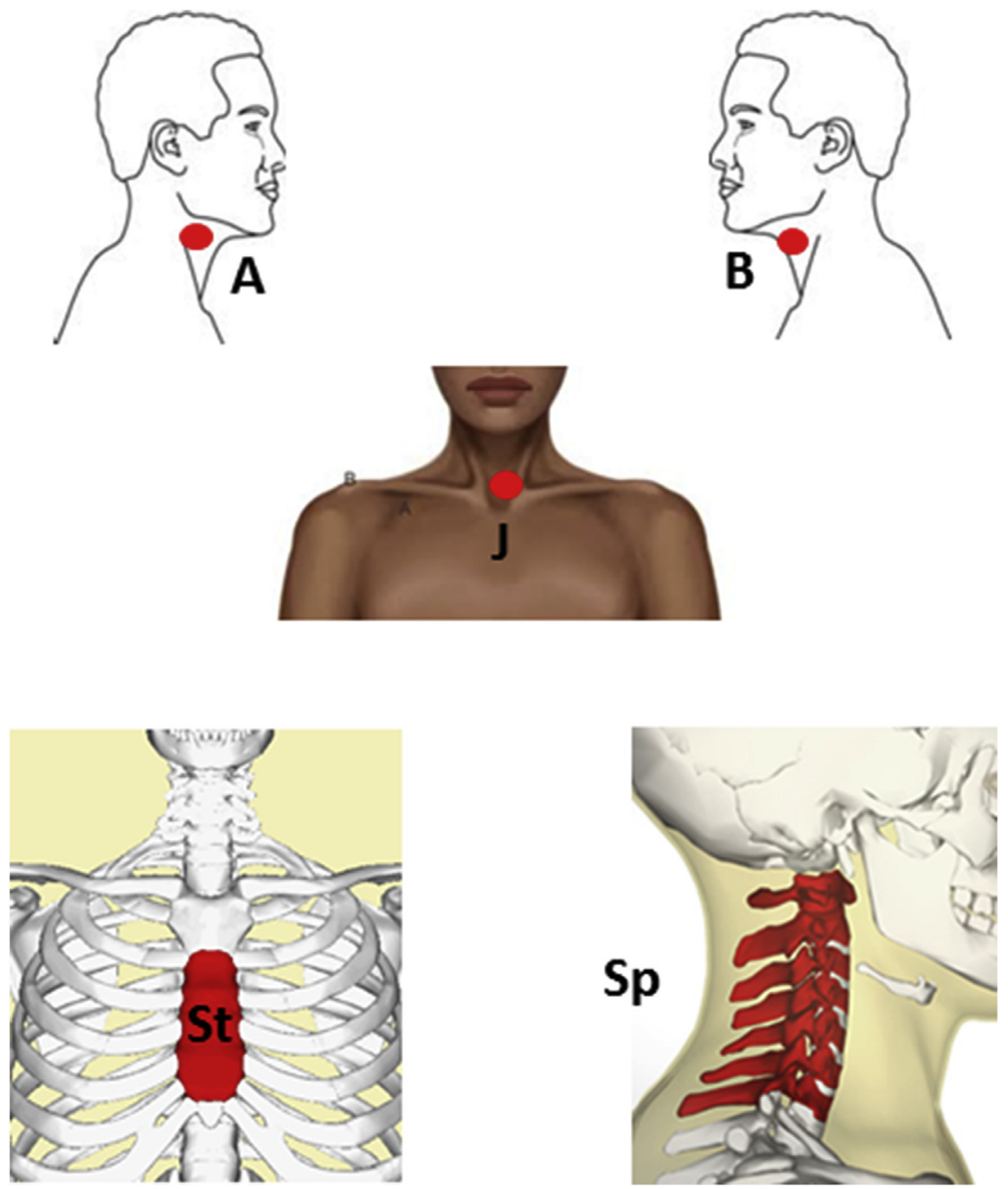

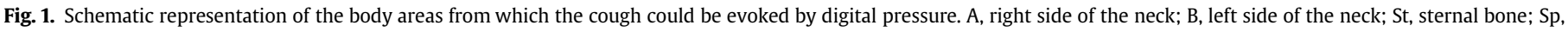
cervical vertebrae. See text for further details.

\section{Discussion}

We are not aware of prior descriptions of coughing precipitated by digital pressure on areas of the neck and chest wall. The cough is an exclusively vagal reflex [8], so that it should not be possible to evoke it from areas devoid of vagal sensory innervation such as the neck and the chest wall. In theory, pressure exerted close to the sternocleidomastoid might involve mechanical stimulation of the vagal trunk in the neck. It is well known that vagal fibres in the neck also convey neural signals from the oesophagus [9], and it may be that, in the patient presented here, oesophageal receptors were sensitised by reflux. In such case, pressure on the nerve trunk might acutely amplify the strength of the afferent signal and precipitate the cough. These considerations, however, cannot be invoked to explain coughing by digital pressure exerted over the sternum and/ or the cervical vertebrae (see Fig. 1). Furthermore, with these latter areas, there is no chance to mechanically stimulate underlying vagally-innervated tissues. Interestingly, some recent lines of evidence $[10,11]$ have suggested that the cough reflex sensitivity is inhibited by chest wall vibration in healthy adults [12], pointing to a reflex inhibitory mechanism originating from skin or bone receptors. The cough modality described here, however, pertains to a patient with chronic cough, and likely represent a case of "allotussia", according with current semantics of cough [12]; nonetheless, the mechanisms sub-serving its origin and neurophysiological basis are largely obscure. The skin and the skeletal muscle are innervated also by thin myelinated and unmyelinated neural fibres [13]. Evidence exists [14] that neural inputs originating from skeletal muscle receptors (and hypothetically skin receptors) release substance P in the nucleus tractus solitarii (NTS). Knowledge of the effect of substance P on the excitability of NTS neurones may provide insight into the neurochemical basis for facilitation and/or activation of the cough reflex following mechanical stimulation of discrete regions of the neck and chest wall in sensitised patients. The finding of a short lasting abolishment of coughing provoked by digital pressure on the cervical spine following application of dry ice points to the possibility of a reflex facilitatory mechanism for cough that can temporarily be silenced by cooling of the skin layer. Further mechanistic and clinical studies are needed to clarify the cough modality described here.

\section{Conflicts of interest}

FL and GAF declare no conflict of interests related to the present paper.

\section{Authorship contributions}

FL and GAF conceived and designed the study, analysed and interpreted the data. Both the Authors wrote the manuscript, and 
the decision to submit it for publication. GAF is the guarantor.

\section{Funding}

This research received no specific grants from any funding agency in the public, commercial, or non-profit sectors.

\section{References}

[1] W.J. Song, Y.S. Chang, S. Faruqi, J.Y. Kim, M.G. Kang, S. Kim, E.J. Jo, M.H. Kim, J. Plevkova, H.W. Park, S.H. Cho, A.H. Morice, The global epidemiology of chronic cough in adults: a systematic review and meta-analysis, Eur. Respir. J. 45 (5) (2015) 1479-1481.

[2] P.G. Gibson, A.E. Vertigan, Management of chronic refractory cough, B.M.J. 14 (2015) h5590.

[3] S.S. Birring, Controversies in the evaluation and management of chronic cough, Am. J. Respir. Crit. Care Med. 183 (6) (2011) 708-715.

[4] M. Shaw, Diagnostic utility of reflux disease symptoms, Gut 53 (suppl4) (2004) $25-27$

[5] F. Lavorini, G.A. Fontana, E. Chellini, C. Magni, M. Pistolesi, J. Widdicombe, Respiratory expulsive efforts evoked by maximal lung emptying, Chest 140 (3) (2011) 690-696.
[6] F. Lavorini, G. Fontana, E. Chellini, C. Magni, M. Pistolesi, J. Widdicombe, The Fontana paradoxical reflex? Chest 140 (3) (2011) 586-588.

[7] F. Lavorini, E. Chellini, F. Bigazzi, E. Surrenti, G.A. Fontana, The clinical value of deflation cough in chronic coughers with reflux symptoms, Chest 149 (6) (2016) 1467-1472.

[8] B.J. Canning, N. Mori, S.B. Mazzone, Vagal afferent nerves regulating the cough reflex, Respir. Physiol. Neurobiol. 152 (3) (2006) 223-242.

[9] M. Hennel, M. Brozmanova, M. Kollarik, Cough reflex sensitization from esophagus and nose, Pulm. Pharmacol. Ther. 35 (2015) 117-121.

[10] T. Kondo, I. Kobayashi, N. Hayama, Y. Ohta, An increase in the threshold of citric acid-induced cough during chest wall vibration in healthy humans, Jpn. J. Physiol. 48 (5) (1998) 341-345.

[11] N. Kashiwazaki, S. Ebihara, P. Gui, N. Katayama, K. Ito, R. Sato, C. Oyama T. Ebihara, M. Kohzuki, Inhibitory effect of cervical trachea and chest wal vibrations on cough reflex sensitivity and perception of urge-to-cough in healthy male never-smokers, Cough 9 (1) (2013) 22.

[12] K.F. Chung, D. Bolser, P. Davenport, G. Fontana, A. Morice, J. Widdicombe, Semantics and types of cough, Pulm. Pharmacol. Ther. 22 (2) (2009) 139-142.

[13] M.P. Kaufman, J.C. Longhurst, K.J. Rybicki, J.H. Wallach, J.H. Mitchell, Effects of static muscular contraction on impulse activity of groups III and IV afferents in cats, J. Appl. Physiol. 55 (1983) 105-112.

[14] J.T. Potts, I.E. Fuchs, J. Li, B. Leshnower, J.H. Mitchell, Skeletal muscle afferent fibres release substance $\mathrm{P}$ in the nucleus tractus solitarii of anaesthetized cats, J. Physiol. 514 (Pt 3) (1999) 829-841. 